\title{
Chronic Headache with Unusual Episode: A Case of Cerebral Venous Sinus and Extracranial Venous Thrombosis in Antiphospholipid Syndrome
}

\author{
Chaw Su Naing ${ }^{*}$, Lim Wan Tin ${ }^{1}$, Slapak Gabrielle ${ }^{2}$, Balakrishnan Tharmmambal ${ }^{1}$ \\ ${ }^{1}$ Department of Internal Medicine, Singapore General Hospital, Singapore City, Singapore \\ ${ }^{2}$ Basildon and Thurrock University NHS Foundation Trust, Essex, UK \\ Email: naing.chaw.su@sgh.com.sg
}

Received 3 September 2014; revised 23 October 2014; accepted 26 November 2014

Copyright (C) 2014 by authors and OALib.

This work is licensed under the Creative Commons Attribution International License (CC BY). http://creativecommons.org/licenses/by/4.0/

(c) (i) Open Access

\begin{abstract}
Cerebral venous sinus thrombosis (CVST) with concomitant extracranial venous thrombosis is not a common clinicopathological entity. Extensive venous thrombosis involving head and neck affects young adult and female gender with isolated CVST contributing about $75 \%$ of the cases. The clinical features of CVST are nonspecific and can mimic various conditions. The neuroimaging investigation especially CT, MR angiography and venography are used to establish the diagnosis. This case highlights the awareness of the diagnostic difficulties, identifying the etiology and initiating effective treatment. The diagnosis of head and neck venous thrombosis is by having a high index of suspicion based on the clinical context and early recognition to lower the associated morbidity and mortality thus improving clinical outcomes in this condition.
\end{abstract}

\section{Keywords}

Headache, Cerebral Venous Thrombosis, Internal Jugular Vein Thrombosis, Antiphospholipid Syndrome

Subject Areas: Internal Medicine, Neurology, Rheumatology

\section{Introduction}

Cerebral venous sinus thrombosis (CVST) is a rare disorder and is commonly seen in young patients. Antiphos-

"Corresponding author. 
pholipid (Hughes) Syndrome (APS) is an heterogeneous disorder characterized clinically by thrombosis in a patient who has persistent antiphospholipid antibodies, and often causes complications in pregnancy, such as miscarriages or stillbirth [1]. In the past, APS has been described in systemic and cerebral thrombotic event both arterial and venous in relatively young age with associated high recurrence rate [2] [3]. However, CVST as an initial clinical presentation is relatively rare in APS. CVST extending to the neck veins involving internal jugular vein (IJV), subclavian vein (SV) to brachiocephalic vein (BCV) associated with APS has not been reported in the literature. The highly variable spectrum of clinical presentation of CVST appears to be the clinical diagnostic challenge in majority of the cases especially complicated by proximal extension. Hence, neurological imaging plays a crucial role especially when the clinical presentation is nonspecific. To achieve a precise diagnosis, it is important for a clinician to be aware of the diverse clinical presentation of the disease and relevant supporting investigations and diagnostic imaging.

\section{Case Report}

A 30 years old Chinese female with a past history of chronic headache since the age of 15 was admitted with one day history of progressive occipital headache. The headache was throbbing in nature and radiated from the neck to the occipital area. Her current symptoms were different in character and intensity compared to her previous headache and was associated with photophobia and phonophobia. She did not have fever, vomiting or neck stiffness. History revealed no prothrombotic risks including consumption of oral contraceptive pill or any previous miscarriages. She denied symptoms of photosensitivity, joint pain and rash. She had no significant investigations done in the past for the headache and it usually responded to analgesia. Nevertheless, she reported an episode of pneumonia a week prior to this admission. There was no family history of malignancy, prothrombotic disorders or autoimmune diseases. On examination, she was apyrexial with a blood pressure of 120/70 $\mathrm{mmHg}$, pulse of 70 beats per minute and normal oxygen saturation. She appeared clinically not toxic with normal Glasgow Coma Scale. The systematic examination was normal with no focal neurological abnormality or signs of meningism. However, there was fullness and tenderness in the left side of the neck.

Full blood count showed a normochromic normocytic anemia and thrombocytopenia. Her haemoglobin was at $11.8 \mathrm{~g} / \mathrm{dL}$ (12 - $16 \mathrm{~g} / \mathrm{dl}), \mathrm{MCV} 86.2$ (78 - $98 \mathrm{FL}), \mathrm{MCH} 30.2$ (27 - $32 \mathrm{PG})$ and platelet of $108 \times 10^{9} / \mathrm{L}(140$ - 440 $\left.\times 10^{9} / \mathrm{L}\right)$. She had neutrophil predominance with a total white cell count at $10.6 \times 10^{9} / \mathrm{L}\left(4-10 \times 10^{9} / \mathrm{L}\right)$. PT and aPTT were within normal limits. ESR was elevated at $60 \mathrm{~mm} /$ hour with a normal CRP and procalcitonin. The HIV screen was negative. She had minimal left lower zone infiltrates on her chest X-ray. In view of sudden onset of severe headache, an unenhanced computed tomography (CT) of the brain was performed at the time of admission in the emergency department as a routine screen, and it was unremarkable for any major bleeding.

Her symptoms and neurological signs were monitored closely and despite adequate analgesia, her headache was still persistent. Hence, CT scan of head was repeated along with CT neck. The scan showed extensive acute thrombosis of left transverse to sigmoid sinus, internal jugular vein, subclavian vein to brachiocephalic vein and left transverse and sphenoid sinuses (Figure 1 \& Figure 2).

To evaluate the extent of the clot, Magnetic resonance imaging (MRI) and magnetic resonance venography (MRV) were performed and there was a thrombus within the left transverse and sphenoid sinuses up to the posterior third of the superior sagittal sinus (Figure 3). The deep cerebral venous system was otherwise patent with no midline shift or parenchymal infarct.

Our foremost clinical diagnosis was venous thrombosis due to anti-phospholipid syndrome. The suspicion of APS was supported by patient profile of young, female with unprovoked acute venous thrombosis in the present of thrombocytopenia. Therapeutic dose of subcutaneous low molecular weight heparin was commenced and overlapped with warfarin.

Autoimmune screen revealed a positive Antinuclear antibodies at $>1 / 800$ titre and double stranded DNA measuring $37.05 \mathrm{IU} / \mathrm{ml}$. Other autoimmune markers including ENA (Extractable nucleic acid) profile, Complement C3, C4, protein S and protein C were normal. Lupus anticoagulant was detected and anti-thrombin III was normal. Both Anticardiolipin IgM and IgG were negative and Factor V Leiden was not detected.

While on treatment patient developed a bilateral VI cranial nerve palsy and papilloedema noted on fundoscopy and it was due to raised intracranial pressure from venous sinus thrombosis. A repeat CT brain showed no new changes but clot resolution (Figure 4). Both the headache and neurological signs resolved gradually. She was discharged with warfarin and was followed up in outpatient clinic. The Lupus anticoagulant was repeated 


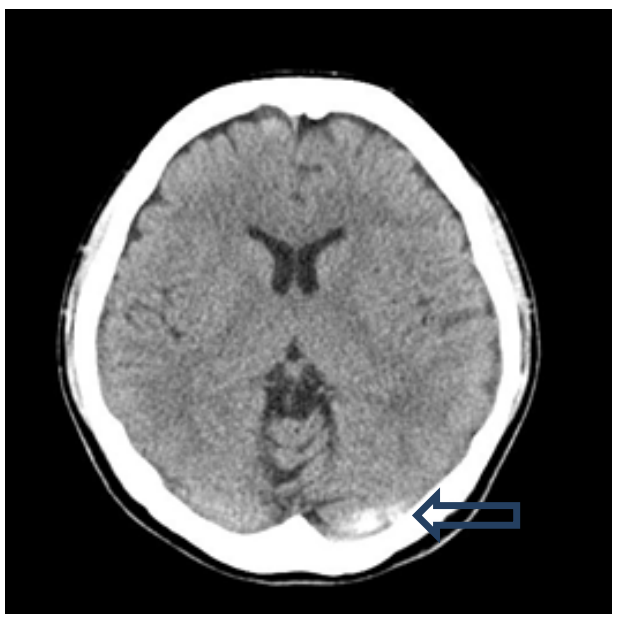

Figure 1. Repeat CT brain showed high density clot in left transverse sinus.

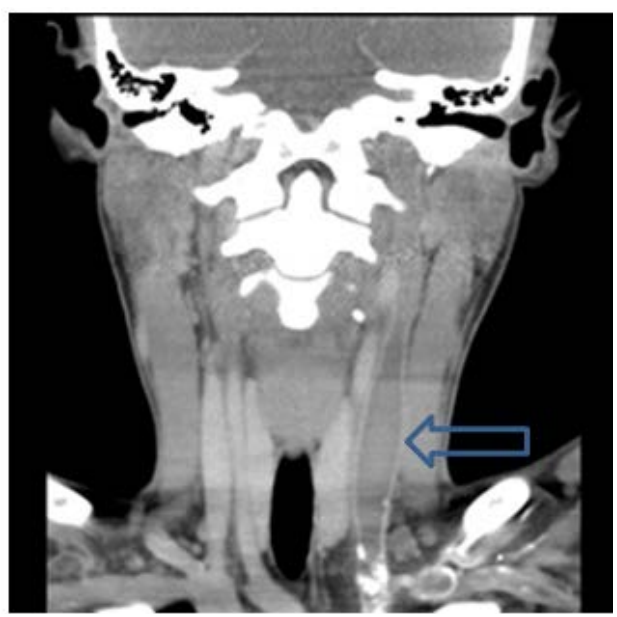

Figure 2. CT neck detected acute venous thrombosis in left internal jugular vein.

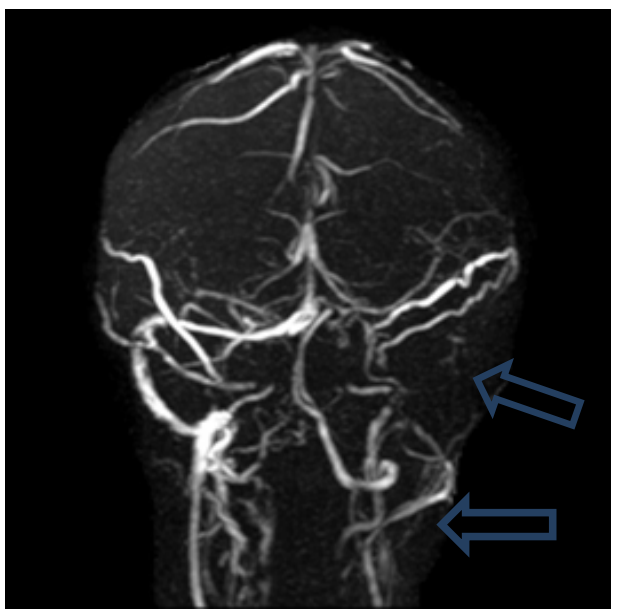

Figure 3. MRV of brain depicted thrombosis in left internal jugular vein, left sigmoid and transverse sinuses with extension of clot. 


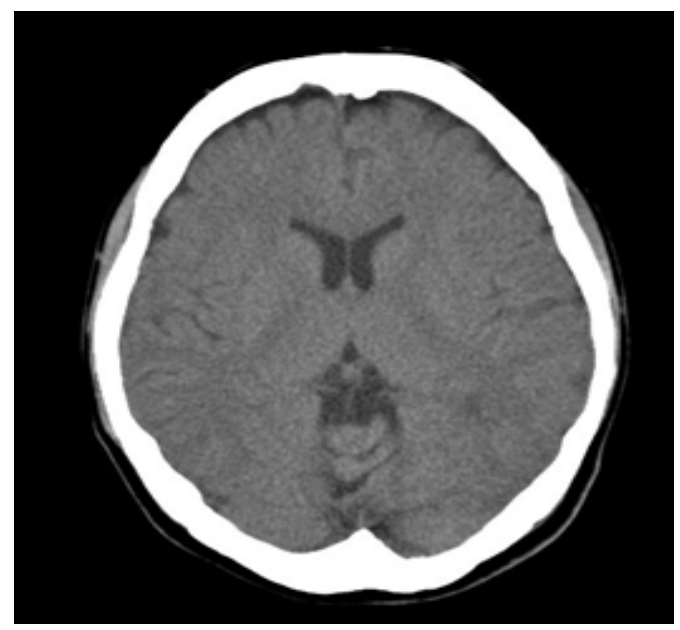

Figure 4. Repeat unenhanced CT brain after 6 days of anticoagulation revealed complete resolution of left transverse sinus clot.

three months later as per guidelines for lupus anticoagulant detection [4], was still demonstrated, confirming the diagnosis of APS.

\section{Discussion}

Simultaneous venous thrombosis involving cerebral veins and internal jugular vein extending intosubclavian and brachiocephalic vein is rare and previously been only described in association with instrumentation, infection and malignancy [5]. IJV thrombosis commonly been identified in cancer, instrumentation and ovarian hyperstimulation syndrome reported in an observational study in France over 9-year period [6]. The common clinical presentation for this would be ipsilateralneck pain and swelling of the affected arm if there is clot extension to subclavian and brachial veins [6].

CVST in itself is a relatively uncommon disorder, with an annual incidence estimated to be about two to four cases per million in the adult population [7] [8]. The etiologies of cerebral venous and sinuses thrombosis can be classified into intracranial infectious and non-infectious types. Genetic, acquired prothrombotic conditions, oral contraceptive usage, pregnancy, post-partum, head injury, tumours, nephrotic syndromes and Behcet's disease have been reported as non-infectious causes of CVST [2]. A multicentre prospective study showed that $0.9 \%$ patients with diagnostic confirmation of APS present with jugular vein thrombosis whilst $0.7 \%$ manifested with CVST [9] with no report on concomitant thrombosis in both. As previously reported in literature and CVST guidelines, the symptoms and clinical course of acute thrombosis in cerebral venous system are highly diverse. The clinical course depends on the site, extent and acuteness of the thrombosis and the presence of collateral circulation from concurrent endogenous thrombolysis or recanalization [7] [9]. The most frequent presentation is headache being the initial symptom and experienced by $75 \%$ - 95\% of patients [7] [10] [11]. The headache has no typical features and invariably associated with other neurological signs including focal deficits, seizure, fluctuating level of consciousness, papilloedema and diplopia (from sixth nerve palsy). Our patient developed both of those features that were well described in previous cases. Isolated headache without focal neurological findings or papilloedema occurs in $23 \%-25 \%$ of patients with cerebral venous thrombosis [10] [11] and it becomes a diagnostic challenge. In such cases, imaging plays an important role in diagnosis. The International Study on Cerebral Vein thrombosis (ISCVT) reported that the median delay from onset of symptoms to hospital admission was 4 days and, from symptom onset to diagnosis was 7 days [12]. The readily available neuroimaging led to diagnostic confirmation in 4 days from onset of symptoms for our case. The clinical spectrum of disease and onset of symptoms are main contributing factors for delay in diagnosis.

Leach et al. described the most common area of thrombosis in as many as $90 \%$ occur particularly in the contiguous transverse and sigmoid sinuses [13] which we identified in our case study. The mechanism of thrombosis in patients with antiphospholipid antibodies is still obscure, although various mechanisms have been described. There were studies elucidating that antiphospholipid antibodies interfere with the endogenous anticoa- 
gulant mechanism via several mechanisms: by disruption of the anticoagulant shield; annexin A5 [14], by inhibition of protein C and its cofactor Protein S [15], by binding and activation of platelets and by interacting with vascular endothelial cells thus inducing expression of adhesion molecules and tissue factor [16]. These effects may or may not depend on beta-2 glycoprotein I [16].

Antiphospholipid antibodies are heterogenous family of Immunoglobulin (Ig) G with or without presence of IgM. There are two subsets within this group namely lupus anticoagulant and Anticardiolipin antibodies and first described in association with non-inflammatory thrombotic endocarditis or with thrombotic venous and arterial occlusions. A systemic review data suggests that Lupus anticoagulant significantly increases the risk of thrombosis [3].

\section{Conclusion}

In conclusion, isolated persistent headache with neck tenderness can be the only and early clinical sign in such thrombosis. A non-contrast cerebral CT is usually performed as initial neuroimaging test to patient with a recent persistent and severe headache in order to rule out a commoner cause of subarachnoid haemorrhage. In CVST, an un-enhanced CT usually shows a hyper dense cortical veins and dural sinuses, however, the initial scan can be abnormal in only about 25\% of cases [7] [13]. Although a plain CT or MRI is useful in the initial evaluation to rule out other causes, a negative scan does not exclude CVST. As in our case, the persistent headache led to the repeat and serial imaging study (either CTV or MRV) that confirmed the diagnosis. High index of suspicion with clinical presentation and patient profile is the key for the diagnosis of this condition leading to early treatment to prevent further complications.

\section{References}

[1] Keeling, D., Mackie, I., Moore, G.W., Greer, I. and Greaves, M., British Committee for Standards in Haematology (2012) Guidelines on the Investigation and Management of Antiphospholipid Syndrome. British Journal of Haematology, 157, 47-58. http://dx.doi.org/10.1111/j.1365-2141.2012.09037.x

[2] Stam, J. (2005) Thrombosis of the Cerebral Veins and Sinuses. New England Journal of Medicine, 352, 1791-1798. http://dx.doi.org/10.1056/NEJMra042354

[3] Galli, M., Luciani, D., Bertolini, G. and Barbui, T. (2003) Lupus Anticoagulants Are Stronger Risk Factors for Thrombosis than Anticardiolipin Antibodies in the Antiphospholipid Syndrome: A Systematic Review of the Literature. Blood, 101, 1827-1832. http://dx.doi.org/10.1182/blood-2002-02-0441

[4] Pengo, V., Tripodi, A., Reber, G., Rand, J.H., Ortel, T.L., Galli, M. and De Groot, P.G., Subcommittee on Lupus Anticoagulant/Antiphospholipid Antibody of the Scientific and Standardisation Committee of the International Society on Thrombosis and Haemostasis (2009) Update of the Guidelines for Lupus Anticoagulant Detection. Subcommittee on Lupus Anticoagulant/Antiphospholipid Antibody of the Scientific and Standardisation Committee of the International Society on Thrombosis and Haemostasis. Journal of Thrombosis and Haemostasis, 7, 1737-1740. http://dx.doi.org/10.1111/j.1538-7836.2009.03555.x

[5] Kulkarni, G.B., Mustare, V. and Varghese, V. (2013) Internal Jugular, Subclavian and Brachiocephalic Vein Thrombosis Associated with Cerebral Venous Sinus Thrombosis. Neurology India, 61, 526. http://dx.doi.org/10.4103/0028-3886.121935

[6] Gbaguidi, X., Janvresse, A., Benichou, J., Cailleux, N., Levesque, H. and Marie, I. (2011) Internal Jugular Vein Thrombosis: Outcome and Risk Factors. QJM, 104, 209-219. http://dx.doi.org/10.1093/qjmed/hcq179

[7] Bousser, M.G. and Ferro, J.M. (2007) Cerebral Venous Thrombosis: An Update. The Lancet Neurology, 6, $162-170$. http://dx.doi.org/10.1016/S1474-4422(07)70029-7

[8] Coutinho, J.M. and Stam, J. (2010) How to Treat Cerebral Venous and Sinus Thrombosis. Journal of Thrombosis and Haemostasis, 8, 877-883. dhttp://dx.doi.org/10.1111/j.1538-7836.2010.03799.X

[9] Cervera, R., Piette, J.C., Font, J., Khamashta, M.A., Shoenfeld, Y., Camps, M.T., et al. (2002) Antiphospholipid Syndrome: Clinical and Immunologic Manifestations and Patterns of Disease Expression in a Cohort of 1,000 Patients. Arthritis \& Rheumatism, 46, 1019-1027. http://onlinelibrary.wiley.com/doi/10.1002/art.10187/full http://dx.doi.org/10.1002/art.10187

[10] Saposnik, G., Barinagarrementeria, F., Brown Jr., R.D., Bushnell, C.D., Cucchiara, B., Cushman, M., et al. (2011) Diagnosis and Management of Cerebral Venous Thrombosis: A Statement for Healthcare Professionals from the American Heart Association/American Stroke Association. Stroke: A Journal of Cerebral Circulation, 42, 1158-1192. http://stroke.ahajournals.org/content/42/4/1158.long http://dx.doi.org/10.1161/STR.0b013e31820a8364 
[11] Cumurciuc, R., Crassard, I., Sarov, M., Valade, D. and Bousser, M. (2005) Headache as the Only Neurological Sign of Cerebral Venous Thrombosis: A Series of 17 Cases. Journal of Neurology, Neurosurgery \& Psychiatry, 76, 1084-1087. http://dx.doi.org/10.1136/jnnp.2004.056275

[12] Ferro, J.M., Canhao, P., Stam, J., Bousser, M.G. and Barinagarrementeria, F., for the ISCVT Investigators (2004) Prognosis of Cerebral Vein and Dural Sinus Thrombosis: Results of the International Study on Cerebral Vein and Dural Sinus Thrombosis (ISCVT). Stroke: A Journal of Cerebral Circulation, 35, 664-670.

http://stroke.ahajournals.org/content/35/3/664.long http://dx.doi.org/10.1161/01.STR.0000117571.76197.26

[13] Leach, J.L., Fortuna, R.B., Jones, B.V. and Gaskill-Shipley, M.F. (2006) Imaging of Cerebral Venous Thrombosis: Current Techniques, Spectrum of Findings, and Diagnostic Pitfalls. Radiographics, 26, 19-41. http://dx.doi.org/10.1148/rg.26si055174

[14] Rand, J., Wu, X., Quinn, A. and Taatjes, D. (2008) Resistance to Annexin A5 Anticoagulant Activity: A Thrombogenic Mechanism for the Antiphospholipid Syndrome. Lupus, 17, 922-930. http://dx.doi.org/10.1177/0961203308095029

[15] Malia, R., Kitchen, S., Greaves, M. and Preston, F. (1990) Inhibition of Activated Protein C and Its Cofactor Protein S by Antiphospholipid Antibodies. British Journal of Haematology, 76, 101-107. http://dx.doi.org/10.1111/j.1365-2141.1990.tb07843.x

[16] Greaves, M. (1999) Antiphospholipid Antibodies and Thrombosis. The Lancet, 353, 1348-1353. http://www.sciencedirect.com/science/article/pii/S0140673698103628 http://dx.doi.org/10.1016/S0140-6736(98)10362-8 